\title{
Attractiveness and activity in Internet communities
}

\author{
Gourab Ghoshal, Petter Holme ${ }^{1}$ \\ Department of Physics, University of Michigan, Ann Arbor, MI 48109, U.S.A.
}

\begin{abstract}
Datasets of online communication often take the form of contact sequences-ordered lists contacts (where a contact is defined as a triple of a sender, a recipient and a time). We propose measures of attractiveness and activity for such data sets and analyze these quantities for anonymized contact sequences from an Internet dating community. For this data set the attractiveness and activity measures show broad power-law like distributions. Our attractiveness and activity measures are more strongly correlated in the real-world data than in our reference model. Effects that indirectly can make active users more attractive are discussed.
\end{abstract}

Key words: Internet community; Network Dynamics; Online Dating PACS: 89.65.-s, 89.75.Hc, 89.75.-k

\section{Introduction}

The Internet has revolutionized science and society in many ways. Studies of human communication networks nowadays deal with data sets of thousands people or more. Gone are the days when one had to rely solely on interview surveys and observational studies. These new and larger data sets open up new possibilitiesone does not only get higher precision in the measurement of network structure, one can also rid the structural quantities of finite size effects that would hinder the prediction of phenomena such as large-scale information spreading. The accessibility of large data sets has also drawn the interest of statistical physicists, habitually working in the large-scale limit, to this traditionally sociological field. The sociologists themselves, naturally, contribute to the development, and make the study of communication networks a thriving interdisciplinary arena. Internet has, of course, changed other aspects of society than science. In the perennial human quest to find a partner, romantic or otherwise, the Internet provides a new modus operandi. In

$\overline{1}$ Corresponding author. Tel.: +1 734647 9568,E-mail: pholme@umich.edu 
this paper we study a Swedish Internet community, pussokram.com, intended for romantic communication among adolescents. The methods we propose do not only work for dating communities, but for Internet communities in general. Our definition of an Internet community is a set of HTML-pages facilitating some type of directed messages (i.e. not all messages are broadcasted to the whole community), and where each member is associated with a home page. This definition includes social networking sites (4) but excludes, for example, news groups (9). The data we use, the same as in Ref. (11), are anonymized lists of contacts-triples $(i, j, t)$ where $i$ is the ID-number of the sender, $j$ is the ID-number of the receiver, and $t$ is the time (in seconds) since an arbitrary start time. The IDs are integer numbers with no relation to the users of the real community. We do not have access to the messages themselves, nor the individual presentations on the user homepages (or any other text or images either).

Researched data sets of electronic communication have often been constructed from email exchange $(1 ; 5 ; 6 ; 15 ; 19 ; 20)$, and to a lesser extent from communication within Internet communities $(8 ; 11 ; 17)$. The advantage with Internet communities is that they are a closed system-all communication can be recorded. The network of emails is a much larger and more important phenomenon. But studies of email networks are plagued by statistical biases due to the openness of the system. For email communication one typically samples a set of individuals and either restricts the data to messages sent within the group (which leaves the message set incomplete) (6) or one includes contacts to outer vertices, which does not include contact between outer vertices. For this reason the study of Internet communities may not only be of interest per se, but is also illuminating towards the general structure of electronic communication.

The goal of this paper is to say something about what makes a user successful in the community. Given our very restricted data-needless to say, the most relevant information is in the text and imagery we do not have-is there anything that can be said? We propose simple measures for attractiveness and activity and observe that they are positively correlated in the contact sequences. More than that, these two quantities are more strongly correlated for the real-world data than for the null model we propose. It thus pays off, directly or (more likely) indirectly, to be an active community member.

\section{The community}

This study is based on data from the Swedish Internet community pussokram.com, logged over 512 days (the same data as in Ref. (11)). Before the start of this data set pussokram.com was a facility to send anonymous e-mails. Our data starts at pussokram.com's beginning as a community. The number of active users grew steadily during the sampling time. At the time of writing, the community form of 
Table 1

The sizes of our three data sets: The number of vertices $N$, edges $M$ and contacts $L$.

\begin{tabular}{l|ccc} 
& $N$ & $M$ & $L$ \\
\hline guest book & 20,683 & 52,547 & 184,325 \\
messages & 21,537 & 50,938 & 264,819 \\
all contacts & 29,341 & 115,684 & 529,890
\end{tabular}

pussokram.com has ceased and the original e-mail service has been re-established. The community form (and its preceding and sequel e-mail service) was targeted at romantic communication among youth; this was written quite explicitly in the administrators' presentation and was conveyed throughout the HTML-pages by text and iconography. This does not mean that all communication was intended to lead to an offline encounter. In fact, a fraction of the communication is probably regular chatting rather than flirting (11).

There are four modes of contacts in the community:

(1) Every user has a publicly accessible "guest book" where other users can post messages.

(2) One can also send direct, e-mail-like, messages.

(3) Each user has a list of "friends" at her (or his) homepage. For user $A$ to be listed as user $B$ 's friend, first $A$ has to send a "friendship request" to $B \ldots$

(4) $\ldots$ and $B$ has to send a "friendship acceptance" to $A$.

In this work we consider the sequences of guest book and message contacts separately and the contact sequence of all four types of contacts taken together. The number of vertices (persons) $N$, edges (non-null dyads, i.e. pairs of vertices between which at least one contact has occurred) $M$ and contacts (communications of one of the four types) $L$ are listed in Table 1.

There are several ways for a member of pussokram.com to find others at the community: 1. At a user homepage other similar users are listed. 2. One can search others based on attributes like gender, interests, place of residence, etc. 3. Pictures of $\sim 50$ users are displayed on the login-page. 4. The "friends" of a user is listed at user homepages. 5. The posters of guestbook messages are displayed. 6. There is a lengthy interview with the "user of the month." A more detailed description of these means of finding others is given in Ref. (11).

\section{Attractiveness and activity measures}

Our measure of attractiveness is the rate of incoming first contacts, i.e. the number of contacts a member $i$ receives from others (s)he have not been in contact with 

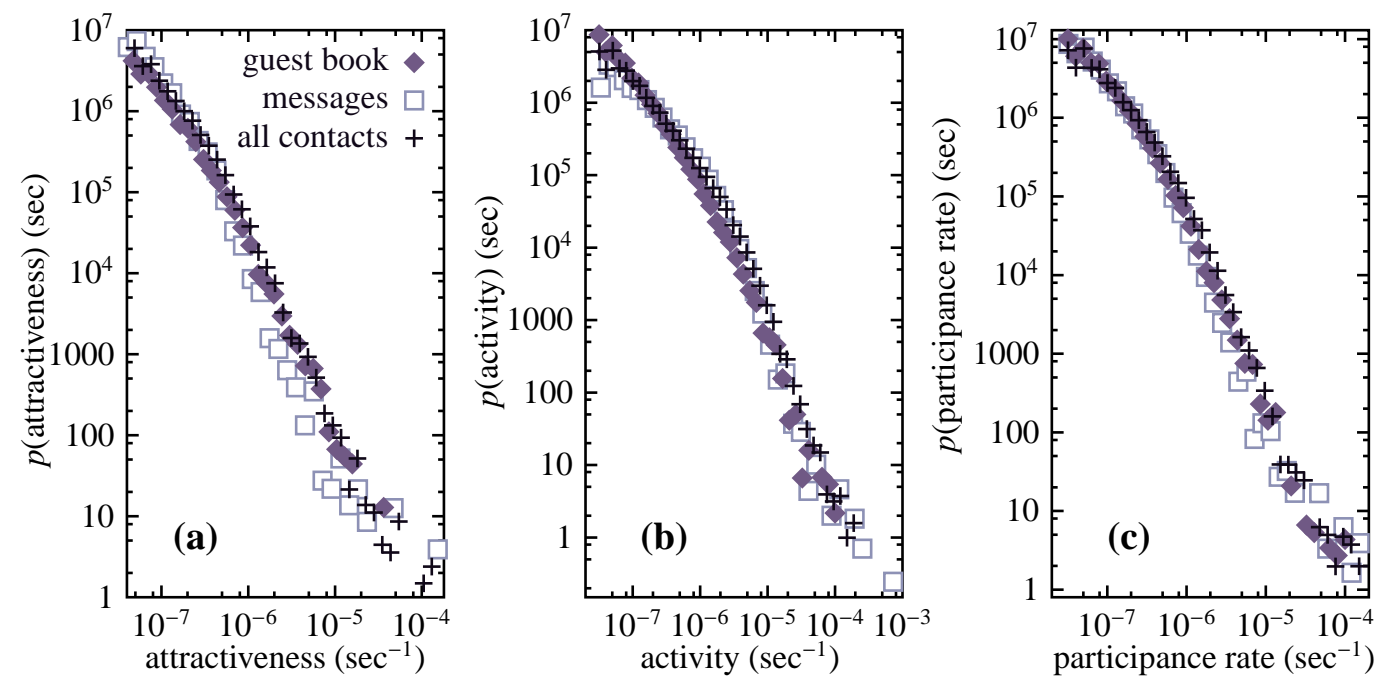

Fig. 1. Logbinned probability density functions of attractiveness (a), activity (b) and participance rate.

before divided by the total time $t(i)$ that $i$ has been present in the data set. How one should determine $t(i)$ in practice is not entirely trivial. One would like $t(i)$ to be the time a vertex is a visible to the rest of the community. The end-time is set by the end of the sampling time frame $t_{\text {end }}$. We chose the starting time for a vertex to be the time (s)he sends or receives the first contact $t_{1}(i)$, so that $t(i)=t_{\text {end }}-t_{1}(i)$. Note that our $t(i)$ is an underestimate of the real presence of a user in the data-one can always register at the community (and thus become visible to others) before sending or receiving any measure. We assume this time is negligible. More elaborate estimates of $t_{1}$ would have to struggle with the fact a member's behavior in the very beginning of his/her career hardly can be estimated from the average behavior. The measure of activity we propose is the rate of any recorded contacts made by a user, i.e. the number of contacts made by a member divided by the same $t(i)$ as above. In addition to our activity and attractiveness measures we also measure the participance ratethe number of other members a member $i$ has contacted, or been contacted by, divided by $t(i)$. This measures how fast an user acquires connections to the rest of the community. Members with a high participance rate will be the hubs of the social network generated by the community interaction.

In Fig. 1 we plot the probability density functions for our three quantities and our three data sets. We observe that all three quantities are highly skewed. A broad distribution of activity has been observed in Usenet communication (22) and was mentioned as a possible cause for the skewed degree distribution in Ref. (11). Golder and Donath (9) discuss the roles of participants in Usenet newsgroups; their classification is partly based on the activity levels. For example, they call the most active users "celebrities," whereas "newbies" (new users) and "lurkers" (users who read but do not post messages) occupy the low-end of the activity spectrum. The fact that the pussokram.com data shows a very broad activity distribution is a sign that it is meaningful to talk about "celebrities" and "lurkers" in this community 

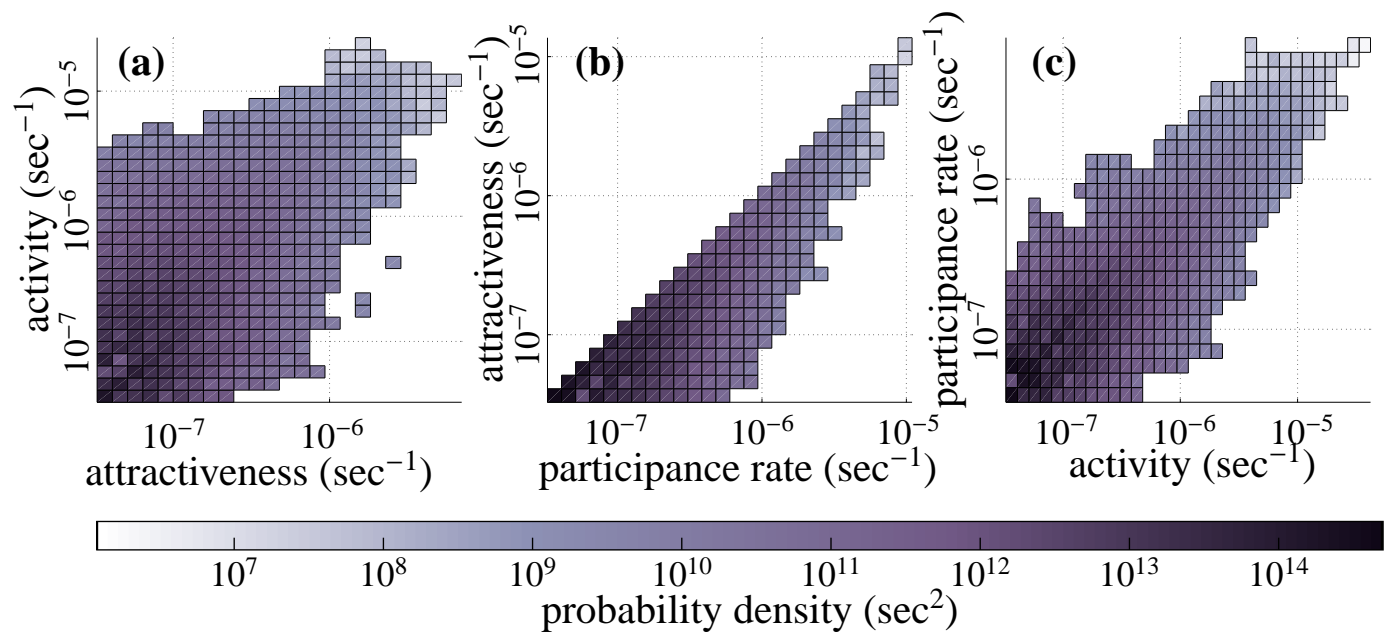

Fig. 2. Logbinned probability density plots for the all-contacts data. (a) shows attractiveness vs. activity; (b) shows activity vs. participance ratio; and (c) shows participance ratio vs. attractiveness.

as well. The fat-tailed distributions of attractiveness and participance rate are less trivial. We will discuss the origins of these and their relation to the skewed activity distribution further below. We note that the functional shape can be described as having two different slopes in the log-log plots of Fig. 1. This is a rather common shape of probability density functions for social and communication network data $(10 ; 11 ; 14)$. We will not dwell further on the details of the functional form.

Our three quantities are not independent. In Fig. 2 we plot probability densities of the three pairs of measures. We see that all three pairs of measures are positively correlated with each other. I.e. an active user is probably also attractive; and has also, likely, a high participance rate. By the definitions of attractiveness and participance rate we note that the attractiveness is strictly smaller than the participance rate, this is evident in Fig. 2(b). The colors of Fig. 2 represent the average of the values of the four corners. Non-zero bins that do not lie on a square with all corners being non-zero will not contribute to these plots. For this reason the largest values of Fig. 1 do not appear in Fig. 2.

\section{Null model}

In the previous section we defined three vertex-specific measures for Internet community contact sequences. We measured the probability distribution and the correlations between the measures. The design of the measures does not exclude that correlations may be induced by the growth of the community. To frame the correlations associated with the psychology of the community members, we need something to compare the values with, i.e. a null model. In fact, we propose two null models, one more and one less restricted. We follow the general approach of Ref. (12) and 
Table 2

The linear correlation coefficients between our quantities for the real-world data and the two null models. Numbers in parentheses are the standard errors in units of the last decimal place. Stars represent the correlation coefficient being significantly lower $(* p<0.05$; ** $p<0.01$ ) for the model networks than for the real-world network (except for the activity vs. participance rate correlations of model 2 where the model values are higher than for the real-world networks). The model network figures are averaged over 100 realizations.

\begin{tabular}{r|ccc} 
real world & attractiveness vs. activity & activity vs. participance rate & part. rate vs. attr. \\
\hline guest book & 0.196 & 0.780 & 0.499 \\
messages & 0.627 & 0.752 & 0.871 \\
all contacts & 0.384 & 0.715 & 0.802 \\
\hline \hline null model 1 & attractiveness vs. activity & activity vs. participance rate & part. rate vs. attr. \\
\hline guest book & $0.059(4)^{*}$ & $0.68(2)$ & $0.1104(7)^{* *}$ \\
messages & $0.057(3)^{* *}$ & $0.71(2)$ & $0.103(3)^{* *}$ \\
all contacts & $0.187(6)^{* *}$ & $0.68(2)$ & $0.279(5)^{* *}$ \\
\hline \hline null model 2 & attractiveness vs. activity & activity vs. participance rate & part. rate vs. attr. \\
\hline guest book & $-0.0417(4)^{* *}$ & $0.9575(2)^{* *}$ & $0.0680(5)^{* *}$ \\
messages & $-0.0263(9)^{* *}$ & $0.9880(2)^{* *}$ & $0.025(2)^{* *}$ \\
all contacts & $-0.072(2)^{* *}$ & $0.9955(3)^{* *}$ & $0.016(3)^{* *}$
\end{tabular}

sample randomizations of the real-world data set rather than constructing parametric null models. In both these models we will keep the sizes $N, M$ and $L$ the same as in the original contact sequences. Not only that, we assume the growth of $N$ is unrelated to the psychology of the community members, we also keep the time evolution of $N$ the same as in the real data. Furthermore, as the community grows with time the communication rate in the data should also be growing. With a parametric null model this would be hard to implement, but we just keep the set of times from the real data and the communication rate exactly the same as in the real-world contact sequence.

In the first, less constrained, model we apply the restrictions above. A randomized contact sequence is constructed as follows:

(1) For each vertex $i$ add one contact to or from (with equal probability) at time $t_{1}(i)$. The other vertex of the contact is chosen with uniform randomness among the vertices present in the data at this time.

(2) Draw $L-N$ times randomly from the set of non-first contacts. (I.e. contacts $(i, j, t)$, such that $t \neq t_{1}(i)$ and $t \neq t_{1}(j)$.) Add contacts between vertices present in the community at these times.

Step 1 ensures that the number of active members is the same in the randomized 
sequence as in the real-world data. Step 2 makes the set of times almost equal to the empirical set of times. Note that, contacts between two vertices $i$ and $j$ at $t=t_{1}(i)=t_{1}(j)$ will give rise to two contacts in the randomized sequence; so the set of non-first contacts used in step 2 will be slightly $(<1 \%)$ larger than $L-N$.

As mentioned in the previous section a broad activity distribution has been observed in many data sets similar to ours. One may argue that activity is a rather independent trait, little connected to the contact dynamics. Whether or not this is true, we construct a null model to test how the reality differs from such a scenario. To generate a random sequence realization for this model we loop over all contacts $(i, j, t)$ and replace $j$ (the vertex the message is sent to) by a random vertex $j^{\prime} \neq i$ present in the community at time $t$. Clearly this procedure conserves $L$. It may result in a few isolated vertices (vertices of zero out-degree who looses their incoming links), and a slightly altered time evolution of $N$ (vertices whose first contact is incoming may appear later in the randomized community than in the real-world data). For our data these effects are small, and we assume them to be negligible.

Values of the linear (Pearson's) correlation coefficient between attractiveness, activity and participance rates for the real and null model networks can be found in Table 2. First we note that the attractiveness and activity are more strongly correlated in the real community than in both models, and that this holds for all three networks. This means that the correlation between attractiveness and activity is not an artifact of the growth of the community (by comparison with model 1), neither is it a result of a skewed distribution of a (hypothetical) intrinsic activity (by comparison with model 2). Unlike the attractiveness vs. activity correlation, the correlation between activity and participance rate seems to a large extent to be an effect of the growth of the network. We see that model 2 induces a very high correlation between activity and participance rate. Model 2 randomizes the recipients but keeps the rest of the communication the same as in the original data. The fact that the users engage in dialogs is an explanation for the lower correlation in the real world data - two users sending contacts to only each other, but frequently is active but have low participance ratio. The correlation between the participance rate and attractiveness of the real data is even more different from the null model than the attractiveness-activity correlation. This means that the vertices of highest degree in the social network generated by the communication are also the one that most frequently get incoming new contacts. Another interesting observation is that the messages data have higher attractiveness correlations than the guest book and all contacts data. Since the messages are not publicly visible one can assume that the real romantic communication takes place here rather than in the guest book writing. This strengthen the conclusion that active users are attractive in Internet dating. We note that (Spearman type) rank correlations are typically a little stronger than the linear correlations, but since they add little new information to the discussion we do not include them in our tables. 


\section{Summary and discussion}

In this paper we have introduced three measures to characterize users in online communities: attractiveness, activity and participance rate (measuring how fast a user get to know others in the community). While these three measures are sensible in general Internet communities, we evaluate them for an Internet dating community (11). How and why people end up as partners is well studied in an off-line setting (2; 3); attractiveness in terms of body shape $(7$; 18), facial characteristics $(16)$ and body odor (21) are all well-known. With Internet communities playing an increasingly important role on the dating scene (8) one need to complement the studies of in-real-life attractiveness with investigations of online romantic preferences. In this paper we find that the attractiveness (the number of incoming new contacts per time) is positively correlated with activity (the number of contacts taken per time), and that this correlation is significantly stronger than for our two null models. We find this heightened correlation in the real-world data even stronger in the dataset of e-mail-like messages than in the other data sets (including messages visible to the whole community). Since the activity, as we measure it, is rather invisible to other members, this correlation has to be a secondary effect. (Writing in guest books increases ones visibility and is a, presumably small, direct effect.) It is natural to assume that the users that send many messages, and thus invest much time into their community, also spend comparatively more time embellishing their homepages. The conclusion is thus that it pays off to spend effort on one's personal presentation. Another factor is that highly active users are most likely logged in more often than low-activity users. Since currently logged in users are displayed, the increased visibility of frequently logged in members may boost the attractiveness of active users. We also find highly skewed, power-law-like distributions of attractiveness, activity and participance rate. The mechanisms behind electronic communication and offline behavior are presumably very different. Nevertheless, similar quantities in off-line relationships, such as the number of partners per time, are also known have this feature (13).

Research on the structure and dynamics of Internet communities is still a young field. Their statistical advantages (being closed systems) compared to e-mail exchange make us anticipate much future work with both data analysis and modeling approaches.

\section{Acknowledgements}

The authors thank Mark Newman for illuminating discussions; Fredrik Liljeros for comments on the manuscript; and Christian Wollter and Michael Lokner for help with the data acquisition. 


\section{References}

[1] L. Adamic and E. Adar. How to search a social network. To appear in Social Networks.

[2] S. S. Brehm. Intimate Relationships. Random House, New York, 1985.

[3] D. M. Buss. The evolution of desire: strategies of human mating. Basic Books, New York, 1994.

[4] J. Donath and D. Boyd. Public displays of connection. BT Technology Journal, 22(4):71-82, 2004.

[5] H. Ebel, L.-I. Mielsch, and S. Bornholdt. Scale-free topology of e-mail networks. Phys. Rev. E, 66:035103, 2002.

[6] J.-P. Eckmann, E. Moses, and D. Sergi. Entropy of dialogues creates coherent structures in e-mail traffic. Proc. Natl. Acad. Sci. USA, 101:14333-14337, 2004.

[7] J. Fan, W. Dai, F. Liu, and J. Wu. Visual perception of male body attractiveness. Proc. R. Soc. B, 272:219-226, 2005.

[8] A. T. Fiore. Romantic regressions: An analysis of behavior in online dating systems. Master's thesis, MIT Media Lab, 2004.

[9] S. A. Golder and J. Donath. Social roles in electronic communities. Sociable Media Group, MIT Media Laboratory.

[10] P. Holme. Network reachability of real-world contact sequences. Phys. Rev. E, 71:046119, 2005.

[11] P. Holme, C. R. Edling, and F. Liljeros. Structure and time evolution of an Internet dating community. Social Networks, 26:155-174, 2004.

[12] L. Katz and J. H. Powell. Probability distributions of random variables associated with a structure of the sample space of sociometric investigations. Ann. Math. Stat., 28:442-448, 1957.

[13] F. Liljeros, C. R. Edling, L. A. N. Amaral, H. E. Stanley, and Y. Åberg. The web of human sexual contacts. Nature, 411:907, 2001.

[14] M. E. J. Newman. Scientific collaboration networks. I. Network construction and fundamental results. Phys. Rev. E, 64:016131, 2001.

[15] M. E. J. Newman, S. Forrest, and J. Balthrop. Email networks and the spread of computer viruses. Phys. Rev. E, 66:035101, 2002.

[16] D. I. Perrett, K. J. Lee, I. S. Penton-Voak, D. R. Rowland, S. Yoshikawa, D. M. Burt, S. P. Henzi, D. L. Castles, and S. Akamatsu. Effects of sexual dimorphism on facial attractiveness. Nature, 394:884-887, 1998.

[17] R. Smith. Instant messaging as a scale-free network. e-print cond-mat/0206378, June 2002.

[18] M. J. Tovée, S. Reinhardt, J. L. Emery, and P. L. Cornelissen. Optimal BMI and maximum sexual attractiveness. Lancet, 399:215-216, 1999.

[19] J. R. Tyler and J. C. Tang. When can I expect an email response? A study of rhythms in email usage. Technical report, Hewlett-Packard Labs, 2003.

[20] J. R. Tyler, D. M. Wilkinson, and B. A. Huberman. Email as spectroscopy: automated discovery of community structure within organizations. In M. Huysman, E. Wenger, and V. Wulf, editors, Communities and Technologies, pages 
81-96. Kluwer, Deventer, 2003.

[21] C. Wedekind and D. Penn. MHC genes, body odours, and odour preferences. Nephrol. Dial. Transplant., 15:1269-1271, 2000.

[22] S. Whittaker, L. Terveen, W. Hill, and L. Cherny. The dynamics of mass interaction. In S. Poltrock and J. Grudin, editors, Proceedings of The 1998 ACM Conference on Computer Supported Cooperative Work, pages 257-264, New York, 1998. Association for computing machinery. 